\title{
Outcome Predictors of SD-OCT-Driven Intravitreal Ranibizumab in Choroidal Neovascularization due to Myopia
}

\author{
Maria-Magdalena Guichard ${ }^{\mathrm{a}}$ Géraldine Peters $^{\mathrm{a}}$ Cengiz Tuerksever ${ }^{\mathrm{a}}$ \\ Christian Pruente ${ }^{b, c}$ Katja Hatz ${ }^{a, b}$ \\ a Vista Klinik, Binningen, Switzerland; ${ }^{\mathrm{b}}$ Department of Ophthalmology, University of Basel, Basel, Switzerland; \\ 'Department of Ophthalmology, Kantonsspital Liestal, Liestal, Switzerland
}

\section{Keywords}

SD-OCT · Ranibizumab · Choroidal neovascularization ·

Myopia $\cdot$ Predictor

\begin{abstract}
Purpose: To analyze the efficacy and outcome predictors of SD-OCT (spectral-domain optical coherence tomography)driven ranibizumab treatment in patients with choroidal neovascularization due to myopia (mCNV). Methods: This prospective investigator-initiated study includes 20 patients with treatment-naïve mCNV. Evaluation included best-corrected visual acuity (BCVA), morphological SD-OCT parameters, and treatment frequency. Results: From baseline to month 12 , BCVA improved from $58.5 \pm 16.9$ to $66.1 \pm 14.9$ letters. Central retinal thickness (CRT) significantly decreased, and qualitative SD-OCT parameters improved. Better baseline visual acuity (VA), lower spherical equivalent, better inner/outer segment line and external limiting membrane integrity showed a significant positive effect on BCVA outcome. Less fluctuation in CRT (worst minus best CRT) indicated better BCVA at 12 months. No serious adverse events occurred. Conclusions: SD-OCT-guided intravitreal ranibizumab treatment in mCNV was efficient and safe. We determined useful predictive factors in regard to VA outcome after 12 months.

(c) 2019 The Author(s)

Published by S. Karger AG, Basel
\end{abstract}

karger@karger.com

www.karger.com/oph

Karger

\section{(C) 2019 The Author(s)}

Published by S. Karger AG, Basel

Karger

Open access

This article is licensed under the Creative Commons AttributionNonCommercial-NoDerivatives 4.0 International License (CC BY NC-ND) (http://www.karger.com/Services/OpenAccessLicense). Usage and distribution for commercial purposes as well as any distribution of modified material requires written permission.

\section{Introduction}

In the United States, myopia affects $24 \%$ of adults aged $>40$ years, equivalent to approximately 34 million people in 2010. This number is predicted to reach approximately 45 million in 2050 [1]. Pathologic myopia (PM) is the second cause of choroidal neovascularization (CNV) after age-related macular degeneration (AMD) and the first cause in patients younger than 50 years. Among patients younger than 50 years, myopia accounted for $62 \%$ of cases of CNV [2]. The natural history of subfoveal myopic CNV ( $\mathrm{mCNV}$ ) has been evaluated in various studies [37]. The precise pathogenesis of CNV in eyes with PM is unclear, although thinning of the choroid and stretching of retinal tissue appear to be involved [6].

The features of CNV in PM are different from other etiologies, such as AMD [8]. Serous retinal detachment is shallower, limited, and may often be difficult to detect with stereoscopic viewing. Retinal hemorrhage, if present, is usually minimal, and hard exudates are not often noted. The total volume of the neurosensory retina is sig-

Maria-Magdalena Guichard and Géraldine Peters contributed equally to this work.

This prospective trial has been registered at ClinicalTrials.gov (NCT03409250). 
nificantly less in patients with $\mathrm{CNV}$ secondary to $\mathrm{PM}$ as compared to patients with CNV secondary to AMD [9, 10]. These findings may reflect reduced exudation associated with $\mathrm{mCNV}$ and thus decreased levels of intraretinal edema in the form of cystic space formation or diffuse "spongy" retinal thickening. Therefore, highly sensitive measurements are needed to detect $\mathrm{mCNV}$ activity. These can be provided using high-resolution OCT (optical coherence tomography) such as SD-OCT (spectral domain) or swept-source OCT, fluorescein angiography (FA), and indocyanine green angiography [11].

The natural long-term visual outcome of $\mathrm{mCNV}$ was found to be extremely poor - the visual acuity (VA) of almost all of the patients dropped to $20 / 200$ or less within 5-10 years [4]. Before the introduction of intravitreal anti-VEGF therapy for CNV, laser photocoagulation, vPDT (verteporfin photodynamic therapy), and surgical excision or macular translocation were performed to treat $\mathrm{mCNV}$. Currently, ranibizumab and aflibercept are licensed anti-VEGF molecules for first-line treatment of $\mathrm{mCNV}$. The phase II REPAIR study showed that ranibizumab was effective in preventing vision loss and improving vision with a median of 3.0 injections over 12 months $[12,13]$. The phase III RADIANCE trial showed that individualized ranibizumab treatment provides superior VA benefits versus vPDT up to month 3. Continued individualized ranibizumab treatment was effective in further improving and sustaining BCVA in patients with mCNV over 12 months [14].

Outcome predictors of anti-VEGF therapy have been evaluated in several common diseases like neovascular AMD (nAMD) and diabetic macular edema. In nAMD, intraretinal cystoid fluid seems to have a negative impact on VA while the presence of subretinal fluid was often associated with a superior visual benefit [15]. Further alterations in the outer retinal layers appear to affect visual outcome in nAMD despite the poor reproducibility of current assessment strategies in many studies [15]. In diabetic macular edema, poor BCVA, submacular fluid, young age, and short diabetes duration were found in patients with very good VA improvement [16]; the height of intraretinal cysts at baseline was a good predictor of functional and anatomical improvement [17]. The RADIANCE subanalysis assessed the impact of different baseline features in mCNV on BCVA at month 12. The median number of injections varied with ethnicity, baseline BCVA, and CNV size, emphasizing the importance of individual treatment in mCNV [18]. Furthermore, chorioretinal atrophy, baseline CNV size, and age were identified as prognostic factors regarding the efficacy of intravitreal injection [19]. Lacquer crack extension to the fovea and peripapillary atrophy were negative prognostic factors of VA improvement [20].

Although the efficacy of intravitreal injection was proven, there is currently no consensus on predictive factors associated with a favorable visual outcome after intravitreal anti-VEGF therapy in $\mathrm{mCNV}$. The aim of this study was to identify outcome predictors of ranibizumab treatment in $\mathrm{mCNV}$.

\section{Patients and Methods}

\section{Patient Selection and Study Conduction}

This is a prospective, 1-arm, monocenter, investigator-initiated study. Twenty treatment-naive patients were included in the study and treated with an intravitreal injection of ranibizumab (0.5 mg; Lucentis ${ }^{\circledR}$; Novartis AG, Basel, Switzerland) at baseline and thereafter on a pro re nata basis following predefined criteria. Two patients were excluded from efficacy analysis ( 1 dropout and 1 major protocol violation); all patients were included in the safety analysis.

Intravitreal injection of $0.5 \mathrm{mg}$ ranibizumab was performed according to standard procedures [21], including topical anesthesia and surface disinfection with $5 \%$ povidone iodine. $\mathrm{mCNV}$ was diagnosed with FA (HRA-2; Heidelberg Engineering, Heidelberg, Germany) using $30^{\circ}$ ETDRS standard settings for central fields. Central retinal structures were evaluated with SD-OCT (Spectralis; Heidelberg Engineering) using the following parameters: horizontal volume scan 19 sections, macular star 6 sections, and horizontal 6-mm scan; high-resolution mode, ART 12. Both qualitative and quantitative assessments were performed according to standard procedures and included central retinal thickness (CRT), central point choroidal thickness (CCT), presence of posterior vitreous detachment (PVD), epiretinal membrane (ERM), macula hole, atrophic dehiscence, atrophy of the pigment epithelium, intraretinal or subretinal fluid, intraretinal hyperreflective foci, external limiting membrane (ELM) disruption, and inner (IS)/outer segment (OS) line disruption within the central $1 \mathrm{~mm}$. Similar to a morphological study in diabetic macular edema [22], IS/OS and ELM disruption (measured by SD-OCT) were evaluated as the means of the horizontal and vertical scans and graded as: 0 (no disruption within the central $1 \mathrm{~mm}$ ), 1 (mild disruption $<1 / 4$ within the central $1 \mathrm{~mm}), 2$ (1/4 to $3 / 4$ disruption within the central $1 \mathrm{~mm}$ ), and 3 (>3/4 disruption within the central $1 \mathrm{~mm}$ ).

During the follow-up period of 12 months, monthly (28 \pm 5 days) ophthalmological examinations, including BCVA, biomicroscopic examination, and SD-OCT assessments were performed. Autofluorescence, FA, and color fundus imaging were performed at baseline and months 3, 6, 9, and 12. Detection of a new CNVrelated hemorrhage on biomicroscopic examination or persisting or new signs of CNV activity at OCT, as defined by intraretinal cysts, subretinal fluid, or fuzzy CNV borders, triggered ranibizumab retreatment considering that any ranibizumab injections can maximally be applied as often as monthly ( $28 \pm 5$ days).

The safety and tolerability of up to monthly dosing of ranibizumab was evaluated by the rates of adverse events and serious adverse events at months 6 and 12 . 
Fig. 1. Efficacy parameters: a Change in best-corrected visual acuity ( $\triangle \mathrm{BCVA})$. b Change in central retinal thickness (CRT)

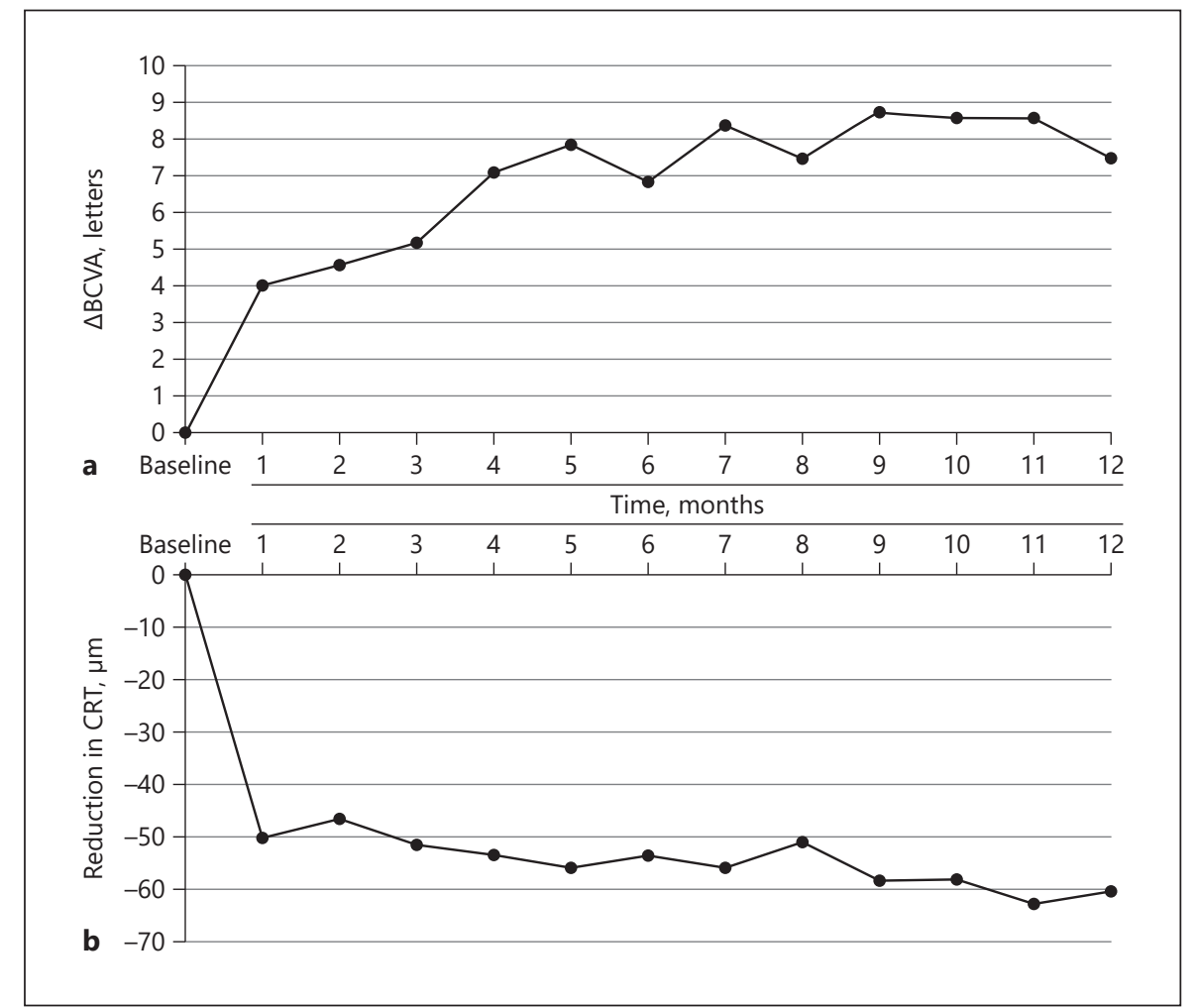

Data Analysis

The variables are expressed as means $\pm \mathrm{SD}$. Shares are shown as percentages. Statistical analyses were carried out with SPSS version 24.0 for Windows (SPSS, Chicago, IL, USA). For comparison of differences to baseline/previous values (dependent variables) and between different subgroups (independent variables) 2 -sided $t$ tests were used. Correlation analyses (univariate) followed Pearson's test. A $p$ value $<0.05$ was considered statistically significant.

\section{Results}

\section{Patient Characteristics}

In this study, 18 treatment naive patients were included in the efficacy analysis. Mean patient age was $65.9 \pm$ 15.3 years. All patients were Caucasian, $77.8 \%$ were female, and $27.8 \%$ had pseudophakia. The baseline spheric equivalent (SE) (before any previous refractive or cataract surgery) was $-14.0 \pm 7.8$ diopters $(\mathrm{dpt})$, range -6.4 to $-31.0 \mathrm{dpt}$.

\section{Efficacy}

VA and Treatment Frequency

The mean baseline BCVA was $58.5 \pm 16.9$ ETDRS letters (Snellen 20/80). The VA gain from baseline to month
12 was $7.6 \pm 11.8$ letters $(p=0.015)$ (Fig. 1a). The largest improvement occurred at month 1 with a gain of $4.1 \pm 7.3$ ETDRS letters $(p=0.031)$. At month 12 there were 2 patients $(11 \%)$ loosing $\geq 15$ ETDRS letters, the proportion of patients gaining $\geq 15$ ETDRS letters was $22 \%$ (4 patients) at 12 months. The mean fluctuation in BCVA during the maintenance phase (individual best minus worst BCVA during months 1-12) was $17.9 \pm 7.0$ letters.

The mean number of injections within 12 months was $3.5 \pm 2.6$ (range $1-9$, median 2.5 ). Four patients (22\%) did not receive any retreatment after the baseline injection. For the remainder, the mean interval from baseline injection to first retreatment was $2.3 \pm 2.2$ months (range 1-8 months).

\section{CNV Activity}

Quantitative SD-OCT Parameters

The mean baseline CRT was $332.4 \pm 63.8 \mu \mathrm{m}$ with a reduction of $-60.4 \pm 56.1 \mu \mathrm{m}$ to month $12(p<0.001)$. The largest reduction occurred after the first month with $-50.1 \pm 51.6 \mu \mathrm{m}(p=0.001)$ and remained stable afterwards (Fig. 1b).

The mean CCT was $75.9 \mu \mathrm{m}$ at baseline with a reduction of $-7.3 \pm 14.6 \mu \mathrm{m}$ to month $12(p=0.049)$. The fluc- 

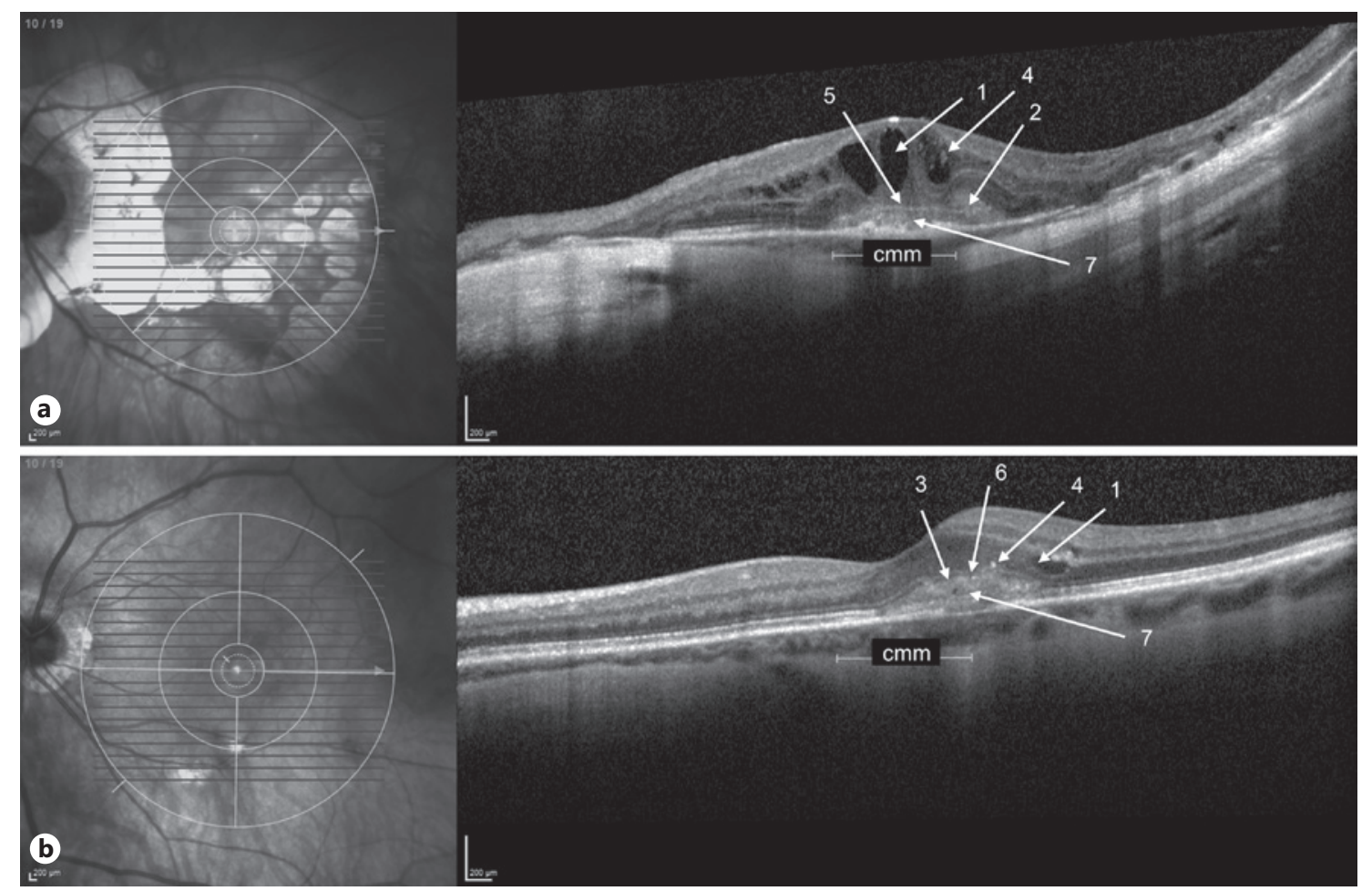

Fig. 2. Typical OCT findings in selected baseline OCT scans of patients 3 (a) and 7 (b), respectively. 1, intraretinal cyst; 2, fuzzy CNV border; 3, sharp CNV border; 4, hyperreflective foci; 5, disruption of external limiting membrane (ELM) and inner/outer segment line; 6, preserved ELM; 7, myopic subretinal neovascularization membrane.

tuation in CCT (individual thickest minus thinnest CCT) was $21.7 \pm 12.7 \mu \mathrm{m}$.

\section{Qualitative SD-OCT Parameters}

The mean percentage of patients with intra- and subretinal fluid was reduced from 55.6 and $50 \%$ at baseline to 16.7 and $11.1 \%$ at month 12 , respectively. At month 12 , $83 \%$ of patients showed no intra- or subretinal fluid in SD-OCT ("dry OCT"). Intraretinal deposits were found in $83.3 \%$ of patients at baseline and in $50 \%$ at month 12 . Fuzziness of the CNV margins was present in $88.9 \%$ at baseline and reduced to $5.6 \%$ at month 12 . PVD was present in 38\% at baseline. There was no difference between eyes with and without PVD at baseline regarding the number of injections $(p=0.365)$. A lamellar macular hole was present in 2 patients (11\%), and a full-thickness macular hole in 1 patient (5.5\%) but remained stable during the study. An ERM was present in 4 patients (22\%) at baseline; there was no difference between eyes with and without ERM at baseline regarding the number of injections $(p=0.262)$. Figure 2 shows OCT images describing typical morphologies.
Table 1. Morphological changes from baseline to month 12

\begin{tabular}{lccr}
\hline & Baseline & Month 12 & $p$ values \\
\hline OCT & & & \\
CRT, $\mu \mathrm{m}$ & 332 & 272 & $<0.001$ \\
CCT, $\mu \mathrm{m}$ & 75.9 & 68.6 & 0.049 \\
Intraretinal cysts, \% & 55.6 & 16.7 & \\
Subretinal cysts, \% & 50.0 & 11.1 & \\
Intraretinal deposits, \% & 83.3 & 50.0 & \\
\hline Fluorescein angiography & & & \\
CNV leakage, \% & 100 & 27.8 & \\
Lesion size, mm ${ }^{2}$ & 1.64 & 0.98 & 0.005 \\
\hline Autofluorescence & & & \\
Central subfield atrophy, \% & 11.1 & 11.1 & \\
\hline Fundus color imaging & & & \\
Hemorrhage, \% & 22.2 & 0 & \\
\hline
\end{tabular}


Table 2. Outcome predictors of visual acuity

\begin{tabular}{lll}
\hline $\begin{array}{l}\text { Predictors at baseline, } \\
\% \text { of patients }\end{array}$ & $\begin{array}{l}\text { BCVA at month 12, } \\
\text { letters }\end{array}$ & $p$ value \\
\hline $\begin{array}{l}\text { BCVA } \\
\quad \geq 55(61 \%) \text { vs. }<55(39 \%) \text { letters }\end{array}$ & $73.3 \pm 12.6 / 54.7 \pm 10.9$ & $\mathbf{0 . 0 0 5}$ \\
$\begin{array}{l}\text { Spheric equivalent } \\
\quad<12(50 \%) \text { vs. } \geq 12(50 \%) \text { dpt. }\end{array}$ & $74.6 \pm 10.1 / 60.8 \pm 16.1$ & $\mathbf{0 . 0 4 8}$ \\
$\begin{array}{l}\text { Inner/outer segment line integrity } \\
\quad \text { Score }<2(28 \%) \text { vs. } \geq 2(72 \%)\end{array}$ & $82.0 \pm 5.7 / 59.9 \pm 12.6$ & $<\mathbf{0 . 0 0 1}$ \\
$\begin{array}{l}\text { External limiting membrane integrity } \\
\quad \text { Score }<2(22 \%) \text { vs. } \geq 2(78 \%)\end{array}$ & $80.7 \pm 5.7 / 61.8 \pm 14.1$ & $\mathbf{0 . 0 0 1}$ \\
$\begin{array}{l}\text { Fuzzy CNV margins } \\
\quad \text { Yes }(89 \%) \text { vs. no }(11 \%)\end{array}$ & 0.555 \\
$\begin{array}{l}\text { Intraretinal fluid } \\
\quad \text { Yes }(56 \%) \text { vs. no }(44 \%)\end{array}$ & $65.3 \pm 15.4 / 72 \pm 11.3$ & 0.227 \\
$\begin{array}{l}\text { Subretinal fluid } \\
\quad \text { Yes }(50 \%) \text { vs. no }(50 \%)\end{array}$ & $70.1 \pm 12.6 / 61 \pm 16.8$ & 0.320 \\
$\begin{array}{l}\text { Central retinal thickness } \\
\quad \geq 330(44 \%) \text { vs. }<330(56 \%) \mu \mathrm{m}\end{array}$ & $69.6 \pm 12.9 / 62.4 \pm 16.7$ & 0.940 \\
\hline
\end{tabular}

Autofluorescence, FA, and Color Fundus Image Parameters

Central subfield pigment epithelium atrophy was detected by autofluorescence in $11.1 \%$ and remained stable during the study. The incidence of leakage at FA was $100 \%$ at baseline and decreased to $27.8 \%$ at month 12 . The lesion size of the CNV decreased significantly from $1.64 \pm$ 1.48 to $0.98 \pm 1.21 \mathrm{~mm}^{2}$ at month $12(p=0.005)$. Bleeding was seen in $22.2 \%$ of eyes at baseline and was absent in all eyes at month 12 at color fundus imaging (Table 1).

Factors Predicting VA and VA Gain at Month 12

We evaluated different baseline characteristics with potential impact on VA or VA gain at month 12 (Table 2). The negative correlation between patient age and BCVA at month 12 did not reach significance (Pearson correlation coefficient $-0.348, p=0.157$ ), but eyes with medium or higher baseline BCVA ( $\geq 55$ letters) showed a significantly better BCVA and greater VA gain at month $12(p=$ 0.005 and $p=0.038)$. Lower baseline SE was associated with a better VA at month $12(p=0.048)$ than a higher baseline SE. Concerning SD-OCT features, better IS/OS line and ELM integrity scores were correlated with a better VA at month $12(p<0.001$ and $p=0.001$, respectively). Less fluctuation in CRT during the maintenance phase (worst minus best CRT) indicated a better visual outcome at month 12 (Pearson correlation coefficient $-0.574, p=0.013)$. Larger baseline lesion size evaluated by FA was correlated with a lower baseline BCVA ( $p=$ 0.01 ) and a lower BCVA outcome at month 12 (Pearson correlation coefficient $-0.490 ; p=0.039$ ). Presence of pseudophakia, subretinal fluid, intraretinal cysts, hyperreflective foci, PVD, ERM, fuzzy CNV margins at SDOCT, pigment epithelium atrophy at autofluorescence, and retinal bleeding at baseline as well as baseline CRT showed no significant association with BCVA outcome or gain at month 12 (for further information, see Table 2).

\section{Discussion}

To attempt a most individualized anti-VEGF treatment, clinicians generally use intravitreal anti-VEGF molecules in a pro re nata regimen to treat $\mathrm{mCNV}$ [11]. The efficiency of intravitreal ranibizumab was proven by several studies $[12,14]$. The functional results of our study are consistent with existing data from Silva et al. [23] using ranibizumab in a prospective 12-month follow-up and showing a significant improvement in VA and a reduction in CRT. In our study, the BCVA gain after 12 months was 7.6 letters after a median of 2.5 injections, which was less than the gain obtained in REPAIR [12] (gain 13.8 letters after a median of 3 injections of ranibizumab) but similar to the results of Silva et al. [23] (gain 8 letters after a mean of 3.6 injections). As in REPAIR, the greatest VA gain was achieved in the first month of treatment. Corresponding to the favorable VA results, CRT showed a significant decrease with the greatest reduction in the first month despite a lower baseline 
CRT in our study compared to REPAIR. This might indicate a less severe macular edema at baseline in our study compared to REPAIR and, therefore, less improvement after treatment.

The association between baseline VA and visual outcome is discussed contrary in mCNV [18-20, 24, 25]. In our study, we found that medium or higher baseline BCVA ( $\geq 55$ letters) predicted a greater VA and a greater BCVA gain at month 12; this might reflect that eyes with better BCVA at baseline had less irreversible retinal damage as well as retinal fibrosis and a better recovery potential. Moon et al. [25] studied the relationship between $\mathrm{mCNV}$ duration and outcome and showed that final BCVA was significantly poorer in cases of poor baseline BCVA, increased age, longer duration of $\mathrm{mCNV}$, and larger baseline CNV size. On the contrary, the RADIANCE subanalysis from Holz et al. [18] showed that patients with high BCVA ( $\geq 73$ letters) at baseline achieved the lowest mean BCVA gain (8.2 letters) at month 12. However, in most analyses evaluating the influence of baseline VA on visual outcome, different thresholds for defining better/worse baseline BCVA were often used due to the limited number of subjects included. This might influence the results significantly.

Similarly to Moon et al. [25], larger baseline FA-evaluated CNV lesion size was a negative predictor for VA at month 12. But, although FA remained the gold standard in the evaluation of retinal damage and leakage for a long time, SD-OCT has attracted more attention in the last years and replaced FA in the follow-up of various retinal diseases. As a consequence, there is growing interest in the use of SD-OCT in the management of mCNV [26] and evaluation of outcome predictors. Within these SD-OCT features, quantitative parameters like absolute CRT seemed not to be correlated to visual outcome in previous studies [26] as well as ours. However, we found that less fluctuation in CRT during the maintenance phase indicated better visual outcome at month 12 as a new aspect. This might reflect that eyes with less severe or no recurrence tend to show a better visual outcome. As described by Introini et al. [26], subretinal fluid and intraretinal fluid are markers of less relevance regarding $\mathrm{mCNV}$ activity. This is also reflected in our study with only about half of the lesions showing intra- or subretinal fluid at baseline, and no effect of the presence of baseline sub- or intraretinal fluid on visual outcome after 12 months.

In our study, IS/OS line integrity was a valuable prognostic marker for visual outcome at month 12 . Several authors have reported that the IS/OS line status corre- lates with VA in several retinal diseases such as retinitis pigmentosa or central serous chorioretinopathy [27, 28 ], and others showed that the IS/OS line appears to be a good predictor of visual outcome in AMD $[29,30]$. Introini et al. [26] extensively studied SD-OCT features correlated with mCNV activity. They found that an "absent or altered" IS/OS junction was associated with CNV activity, but they did not conclude that this feature might predict the treatment outcome in $\mathrm{mCNV}$. The detailed grading of the IS/OS line status within the central $1 \mathrm{~mm}$ might have supported a more detailed analysis and new results in our study. However, grading of outer retina details remains difficult, and, very recently, ELM integrity grading in $\mathrm{mCNV}$ attracted attention [31]. The best approach to grade ELM integrity would of course be its automated full delineation, which would allow a continuous variable in comparison to our ordinal model. In our study, we showed that better ELM integrity within the central $1 \mathrm{~mm}$ was a positive outcome predictor for VA at month 12. Using the above-described (see Methods) grading system, not only patients with complete ELM integrity but also those with less severe disruptions were included in the group of "better ELM integrity" and showed better visual outcomes than those with severe disruptions. In a retrospective analysis with variable follow-up time, Milani et al. [32] reported that any ELM disruption was present in $68 \%$ of eyes at baseline, which was reduced to $41 \%$ during follow-up. Complete integrity of lesion-adjacent ELM and of lesion-adjacent ellipsoid zone at baseline seemed to be factors for better final BCVA.

Concerning the age of patients as predicting factor, significance was not reached in the study. However, our results tended in the direction described by the review of Karagiannis et al. [33]: younger patients tended to have a better visual outcome than older patients. However, we carefully used this information knowing that Leveziel et al. [34] showed that mCNV occurring in older patients ( $>55$ years) were larger than those seen in younger patients, and the better visual outcome in younger patients might also reflect this situation.

This prospective study evaluated a greater variety in possible biomarkers regarding the outcome of intravitreal ranibizumab injections in treatment-naïve mCNV. For image assessments, standardized procedures, including a grading system for IS/OS and ELM disruption, were used. Limitations of the current study are the low number of patients studied, the lack of a control group, and the short follow-up time of 12 months. 
In conclusion, this study shows that SD-OCT-guided intravitreal ranibizumab treatment in $\mathrm{mCNV}$ is efficient and safe, and a variety of predictive biomarkers could be identified. Medium or higher baseline VA, lower baseline SE, less fluctuation in CRT during the maintenance phase, better baseline IS/OS line and ELM integrity, and a smaller lesion size were shown to be correlated with better visual outcome at 12 months. This might indicate that not only early treatment but also prevention of severe recurrences is crucial in terms of visual outcome. The described biomarkers might help to improve the understanding of disease mechanisms and might provide guidance for an individualized treatment plan to achieve optimal functional outcome and disease management in $\mathrm{mCNV}$.

\section{Acknowledgements}

The authors thank Novartis Switzerland for funding this investigator-initiated study in part as well as open access funding. Novartis Switzerland had no influence on the study or the content of the manuscript. The authors further thank Mrs. Christine Knodel for the preparation and evaluation of imaging material, study coordination, and data collection, Mrs. Susanne Müller is acknowledged for her valuable support during data collection and preparation of the manuscript, and Mrs. Anja Burgherr for data monitoring.

\section{Statement of Ethics}

The study followed the tenets of the Declaration of Helsinki and ICH-GCP, and approval was received from the local ethics commission (Ethikkommission Nordwestschweiz, EKNZ; No. 114/10) and health authority (Swissmedic No. 2011DR2095).

\section{Disclosure Statement}

This study was supported in part by Novartis Switzerland; further Novartis Switzerland also provided open access funding but had no influence on the study or the content of the manuscript. Prof. Pruente and Dr. Hatz independently received financial compensation from Novartis Switzerland, Bayer Switzerland, Alcon, Allergan, and Roche for consultancies and contract research. None of the authors reports other financial interests in terms of the presented study.

\section{References}

1 National Eye Institute. Myopia statistics and data, Available from:http://www.nei.nih.gov/ eyedata/myopia.asp\#4. Accessed November 4, 2014.

2 Cohen SY, Laroche A, Leguen Y, Soubrane G, Coscas GJ. Etiology of choroidal neovascularization in young patients. Ophthalmology. 1996 Aug; 103(8):1241-4.

3 Miller DG, Singerman LJ. Natural history of choroidal neovascularization in high myopia. Curr Opin Ophthalmol. 2001 Jun;12(3): $222-4$.

4 Yoshida T, Ohno-Matsui K, Ohtake Y, Takashima T, Futagami S, Baba T, et al. Longterm visual prognosis of choroidal neovascularization in high myopia: a comparison between age groups. Ophthalmology. $2002 \mathrm{Apr}$; 109(4):712-9.

5 Ohno-Matsui K, Yoshida T, Futagami S, Yasuzumi K, Shimada N, Kojima A, et al. Patchy atrophy and lacquer cracks predispose to the development of choroidal neovascularisation in pathological myopia. Br J Ophthalmol. 2003 May;87(5):570-3.

6 Hotchkiss ML, Fine SL. Pathologic myopia and choroidal neovascularization. Am J Ophthalmol. $1981 \mathrm{Feb} ; 91(2): 177-83$.

7 Avila MP, Weiter JJ, Jalkh AE, Trempe CL, Pruett RC, Schepens CL. Natural history of choroidal neovascularization in degenerative myopia. Ophthalmology. 1984 Dec;91(12): 1573-81.

8 Soubrane G. Choroidal neovascularization in pathologic myopia: recent developments in diagnosis and treatment. Surv Ophthalmol. 2008 Mar-Apr;53(2):121-38.

9 Keane PA, Liakopoulos S, Chang KT, Heussen FM, Ongchin SC, Walsh AC, et al. Comparison of the optical coherence tomographic features of choroidal neovascular membranes in pathological myopia versus age-related macular degeneration, using quantitative subanalysis. Br J Ophthalmol. 2008 Aug; 92(8):1081-5.

10 Keane PA, Liakopoulos S, Ongchin SC, Heussen FM, Msutta S, Chang KT, et al. Quantitative subanalysis of optical coherence tomography after treatment with ranibizumab for neovascular age-related macular degeneration. Invest Ophthalmol Vis Sci. 2008 Jul; 49(7):3115-20.

11 Ohno-Matsui K, Ikuno Y, Lai TY, Gemmy Cheung CM. Diagnosis and treatment guideline for myopic choroidal neovascularization due to pathologic myopia. Prog Retin Eye Res. 2018 Mar;63:92-106.

12 Tufail A, Narendran N, Patel PJ, Sivaprasad S, Amoaku W, Browning AC, et al. Ranibizumab in myopic choroidal neovascularization: the 12-month results from the REPAIR study. Ophthalmology. 2013 Sep;120(9): 1944-5.e1.

13 Tufail A, Patel PJ, Sivaprasad S, Amoaku W, Browning AC, Cole M, et al. Ranibizumab for the treatment of choroidal neovascularisation secondary to pathological myopia: interim analysis of the REPAIR study. Eye (Lond). 2013 Jun;27(6):709-15.
14 Wolf S, Balciuniene VJ, Laganovska G, Menchini U, Ohno-Matsui K, Sharma T, et al.; RADIANCE Study Group. RADIANCE: a randomized controlled study of ranibizumab in patients with choroidal neovascularization secondary to pathologic myopia. Ophthalmology. 2014 Mar;121(3):682-92.e2.

15 Schmidt-Erfurth U, Waldstein SM. A paradigm shift in imaging biomarkers in neovascular age-related macular degeneration. Prog Retin Eye Res. 2016 Jan;50:1-24.

16 Sophie R, Lu N, Campochiaro PA. Predictors of Functional and Anatomic Outcomes in Patients with Diabetic Macular Edema Treated with Ranibizumab. Ophthalmology. $2015 \mathrm{Jul}$; 122(7):1395-401.

17 Gerendas BS, Prager S, Deak G, Simader C, Lammer J, Waldstein SM, et al. Predictive imaging biomarkers relevant for functional and anatomical outcomes during ranibizumab therapy of diabetic macular oedema. Br J Ophthalmol. 2018 Feb;102(2):195-203.

18 Holz FG, Tufail A, Leveziel N, Lai TY, Lanzetta P, Wong TY, et al.; RADIANCE Study Group. Ranibizumab in Myopic Choroidal Neovascularization: A Subgroup Analysis by Ethnicity, Age, and Ocular Characteristics in RADIANCE. Ophthalmologica. 2016;236(1): $19-28$.

19 Wang J, Kang Z. Summary of prognostic factors for choroidal neovascularization due to pathological myopia treated by intravitreal bevacizumab injection. Graefes Arch Clin Exp Ophthalmol. 2012 Dec;250(12):1717-23. 
20 Yoon JU, Kim YM, Lee SJ, Byun YJ, Koh HJ. Prognostic factors for visual outcome after intravitreal anti-VEGF injection for naive myopic choroidal neovascularization. Retina. 2012 May;32(5):949-55.

21 Grzybowski A, Told R, Sacu S, Bandello F, Moisseiev E, Loewenstein A, et al.; Euretina Board. 2018 Update on Intravitreal Injections: Euretina Expert Consensus Recommendations. Ophthalmologica. 2018;239(4): 181-93.

22 Hatz K, Ebneter A, Tuerksever C, Pruente C, Zinkernagel M. Repeated Dexamethasone Intravitreal Implant for the Treatment of Diabetic Macular Oedema Unresponsive to AntiVEGF Therapy: Outcome and Predictive SDOCT Features. Ophthalmologica. 2018;239 (4):205-14.

23 Silva RM, Ruiz-Moreno JM, Rosa P, Carneiro A, Nascimento J, Rito LF, et al. Intravitreal ranibizumab for myopic choroidal neovascularization: 12-month results. Retina. 2010 Mar;30(3):407-12.

24 Yang HS, Kim JG, Kim JT, Joe SG. Prognostic factors of eyes with naïve subfoveal myopic choroidal neovascularization after intravitreal bevacizumab. Am J Ophthalmol. 2013 Dec; 156(6):1201-1210.e2.
25 Moon BG, Cho AR, Lee J, Kim YJ, Lee JY, Kim JG, et al. Improved Visual Outcome and Low Recurrence with Early Treatment with Intravitreal Anti-Vascular Endothelial Growth Factor in Myopic Choroidal Neovascularization. Ophthalmologica. 2017;237(3):128-38.

26 Introini U, Casalino G, Querques G, Gimeno AT, Scotti F, Bandello F. Spectral-domain OCT in anti-VEGF treatment of myopic choroidal neovascularization. Eye (Lond). 2012 Jul;26(7):976-82.

27 Witkin AJ, Ko TH, Fujimoto JG, Chan A, Drexler W, Schuman JS, et al. Ultra-high resolution optical coherence tomography assessment of photoreceptors in retinitis pigmentosa and related diseases. Am J Ophthalmol. 2006 Dec;142(6):945-52.

28 Piccolino FC, de la Longrais RR, Ravera G, Eandi CM, Ventre L, Abdollahi A, et al. The foveal photoreceptor layer and visual acuity loss in central serous chorioretinopathy. Am J Ophthalmol. 2005 Jan;139(1):87-99.

29 Sayanagi K, Sharma S, Kaiser PK. Photoreceptor status after antivascular endothelial growth factor therapy in exudative age-related macular degeneration. Br J Ophthalmol. 2009 May;93(5):622-6.
30 Türksever C, Prünte C, Hatz K. Baseline Optical Coherence Tomography Findings as Outcome Predictors after Switching from Ranibizumab to Aflibercept in Neovascular AgeRelated Macular Degeneration following a Treat-and-Extend Regimen. Ophthalmologica. 2017;238(3):172-8.

31 Ding X, Zhan Z, Sun L, Yang Y, Li S, Zhang A, et al. Retinal pigmental epithelium elevation and external limiting membrane interruption in myopic choroidal neovascularization: correlation with activity. Graefes Arch Clin Exp Ophthalmol. 2018 Oct;256(10): 1831-7.

32 Milani P, Pellegrini M, Massacesi A, Moschini S, Setaccioli M, Soranna D, et al. Is ellipsoid zone integrity essential for visual recovery in myopic neovascularization after anti-VEGF therapy? Graefes Arch Clin Exp Ophthalmol. 2017 Sep;255(9):1713-20.

33 Karagiannis D, Kontadakis GA, Kaprinis K, Giarmoukakis A, Georgalas I, Parikakis EA, et al. Treatment of myopic choroidal neovascularization with intravitreal ranibizumab injections: the role of age. Clin Ophthalmol. 2017 Jun;11:1197-201.

34 Leveziel N, Caillaux V, Bastuji-Garin S, Zmuda M, Souied EH. Angiographic and optical coherence tomography characteristics of recent myopic choroidal neovascularization. Am J Ophthalmol. 2013 May;155(5):913-9. 